\title{
REPRÉSENTATIONS MÉCANIQUES ET IMAGINAIRE MÉDIÉVAL
}

Fabienne Pomel

l'ingéniosité humaine et qui suscitent l'admiration par leurs pouvoirs ou effets spectaculaires: machines et projectiles de guerre, pièges en vénerie, mécanismes et artifices destinés à servir l'illusion théâtrale (trappes, mises à feu, mécanismes élévateurs, etc.), ou encore automates dotés de mouvement et mécaniques subtiles comme l'horloge ${ }^{1}$. Ces objets demandent à être resitués dans une histoire des arts mécaniques, marquée par une grande évolution entre les $\mathrm{XII}^{\mathrm{e}}$ et $\mathrm{XV}^{\mathrm{e}}$ siècles, mais aussi à être articulés à une esthétique du spectaculaire et de l'illusion, présente dans le merveilleux romanesque ou la mise en scène théâtrale, notamment celle des mystères.

Les machines ne sont qu'une des catégories d'objets qui relèvent des arts mécaniques, entendus au Moyen Âge comme «l'ensemble des connaissances qui président à la motricité productive du corps, qu'elle soit manuelle ou buccale, ouvrière, ou disons-le, "artistique" ", selon Serge Lusignan ${ }^{2}$. La frontière entre les domaines de savoir-faire et entre le domaine artisanal et artistique n'est pas tracée. En outre, ces savoirs techniques restent en bonne partie absents de la produc-

1. L'horloge a été étudiée dans un précédent programme du CETM : voir Cloches et horloges dans les textes médiévaux. Mesurer et maîtriser le temps, F. Pomel (dir.), Rennes, Presses Universitaires de Rennes, Interférences, 2012

2. "La lettre et le travail: l'impossible point de rencontre des arts mécaniques au Moyen Âge ", dans Le Travail au Moyen Âge. Une approche interdisciplinaire, Louvain-La-Neuve, 1990 (Actes du colloque international des 21-23 mai 1987, éd. J. Hamesse et C. Muraille-Samaran, p. 129-139 (132). 
tion écrite: "Les arts mécaniques circonscrivent des champs de la connaissance maintenus en dehors du savoir organisé de l'École et où le De diversibus artibus associé au nom de "Theophilus" demeure pour longtemps un hapax", note encore S. Lusignan ${ }^{3}$, qui relève l'absence d'autorité ou leur inadéquation au monde médiéval. V. Dominguez note dans le même sens qu'il n'y a pas de description de la fabrication ou du fonctionnement des machines théâtrales, qui relèvent d'un savoir-faire professionnel mais ne trouvent pas leur raison d'être dans les écrits, soit pour des raisons de discrétion, soit parce que la finalité des textes n'implique pas de tels détails. La place des arts dits mécaniques évolue et fluctue aussi pendant la période médiévale dans le sens d'une revalorisation à partir du XII ${ }^{\mathrm{e}}$ siècle: Rupert de Liège (ou de Deutz) ou Honorius d'Autun tentent de les intégrer à un système du savoir, notamment en les rattachant aux dons de l'intelligence. Les arts mécaniques, liés au monde de la matière, forment un septénaire parallèle à celui des arts libéraux chez Hugues de Saint-Victor, qui semble le premier à « attribuer aux arts mécaniques le statut d'une science ${ }^{4}$ ». Hugues de Saint-Victor définit ainsi de manière très large les arts mécaniques comme ce " qui ordonne les activités de cette vie » ou « la science qui regroupe la fabrication de toutes les choses 5 » et inclut ainsi différents domaines de la technique:

La mécanique contient sept sciences: la fabrication de la laine, l'armement, la navigation, l'agriculture, la chasse, la médecine, le théâtre. Trois d'entre elles concernent la protection de la nature vis-à-vis de l'extérieur, ce grâce à quoi la nature se met à l'abri des désagréments. Quatre touchent à la protection interne et font que la nature se nourrit en s'alimentant et en s'entretenant. Il y a là une ressemblance avec le trivium et le quadrivium, puisque le trivium traite des mots, qui sont à l'extérieur, et le quadrivium des concepts qui sont formés à l'intérieur. Voilà les sept servantes que Mercure a reçues en dot de Philologie, tant il est vrai que toute action humaine est au service de l'éloquence à laquelle a été associée la sagesse $^{6}[\ldots]$.

M. Lemoine, traducteur du Didascalicon, note la réhabilitation ainsi opérée: " on peut penser que cette répartition 3+4 rappelant le trivium, était destinée à faire admettre plus facilement l'irruption, au milieu des sciences nobles, de la mécanique, dont la dignité se retrouvait en quelque sorte rehaussée par cette présentation septénaire. Il faut dire que les arts mécaniques, c'est-à-dire en fait

3. Art. cit., p. 129.

4. Piotr Skubiszewski, "L'intellectuel et l'artiste face à l'œuvre à l'époque romane ", dans Le Travail au Moyen Âge, op. cit., p. 263-321, particulièrement "les artes mechanicae et le savoir» (p. 304).

5. Op. cit., respectivement p. 90 et 115.

6. Hugues de Saint-Victor, L'Art de lire. Didascalicon, trad. Michel Lemoine, Éditions du Cerf, 1991, p. 114 (chap. 20). 
la technique, étaient déconsidérés depuis l'Antiquité ${ }^{7}$ ». Frédéric Duval observe que ce schéma dérive d'Augustin et qu' " au XIII ${ }^{\mathrm{e}}$ siècle, plusieurs classifications des sciences tenteront d'opérer une synthèse des schémas augustinien et aristotélicien ${ }^{8}$ ». Il note l'éclipse des arts mécaniques qui concernent «les vilains » au profit des arts de la guerre et de la chasse, plus nobles, ainsi que l'émancipation de la médecine de cette série. Le schéma aristotélicien, à partir d'une axiologie fondée sur le rapport à la nature, promeut plutôt une tripartition entre sciences théoriques, pratiques et productives, la fabrication d'objets se rattachant à la dernière catégorie. Théorie, pratique, mécanique et logique y sont autant d'aspects de la science voire de la sagesse.

Pourtant, au-delà de ces classements savants qui tentent de promouvoir un savoir-faire technique, s'exprime à l'inverse une méfiance envers les arts mécaniques. S. Lusignan l'attribue au rôle central du corps: "En tant que catégorie épistémologique, ils circonscrivent des connaissances extra-textuelles qui n'ont d'existence que pas la médiation du corps de l'ouvrier et c'est visiblement là une source de malaise de la philosophie à leur égard ${ }^{9}$. " On pourrait ajouter aux raisons de cette méfiance la manipulation de la matière sur le principe d'une imitation de la nature et du créateur. La définition avancée par Hugues de Saint-Victor mettait d'ailleurs en avant cette connotation négative à travers une fausse étymologie à partir de moechari (commettre l'adultère), répétée deux fois, qui souligne comme le relève P. Skubiszewski, « le caractère illégitime et imparfait des occupations propres aux artes mechanicae ${ }^{10} »$ :

[...] c'est fort à propos que l'œuvre de l'homme, qui n'est pas la nature mais qui l'imite, est appelée " mécanique ", c'est-à-dire " adultérine ", de même qu'une clef utilisée subrepticement est dite "mécanique 11 ".

On appelle ces sciences mécaniques c'est-à-dire "adultérines ", parce qu'elles traitent de l'œuvre de l'artisan, qui emprunte sa forme à la nature. De même que les sept autres ont été appelées « libérales " soit parce qu'elles exigent des esprits libres, c'est-à-dire dispos et exercés, puisqu'elles débattent des causes des choses, soit parce que dans l'Antiquité, seuls s'y consacraient les hommes libres, c'est-à-dire nobles, tandis que les plébéiens et les garçons des familles non nobles s'adonnaient aux sciences mécaniques, à cause de leur savoir-faire ${ }^{12}$.

7. Introduction à Hugues de SAINT-Victor, L'Art de lire, p. 23.

8. Frédéric Duval, III. Lectures scientifiques, dans Lectures françaises de la fin du Moyen Âge. Petite anthologie commentée de succès littéraires, Droz, 2007, p. 195-198 (p. 196).

9. Article cité, p. 131.

10. Art. cit., p. 300.

11. Op. cit., p. 83 (chap. 9).

12. Op. cit., p. $114-115$ (chap. 20). 
Les arts mécaniques ne sont donc pas sans ambiguïté: l'œuvre de l'artisan emprunte sa forme à la nature et produit une imitation au troisième degré par rapport à la création de Dieu. Toute machine en tant que produit de l'artifice humain, fait de son fabriquant un potentiel rival du Dieu créateur par sa démarche d'imitation de la nature, voire sa prétention à la dépasser. De plus, l'œuvre artisanale à la fois manifeste et tente de réparer les effets de la chute:

L'œuvre de l'artisan consiste à réunir ce qui est séparé ou à séparer ce qui est uni, d'où la formule: «ils se cousirent des ceintures " ${ }^{13}$.

Les machines invitent donc à s'interroger sur la place et la représentation de la mécanique, conçue cette fois au sens moderne, dans la culture médiévale et plus spécialement dans les textes littéraires. Nous avons donc essayé de mener une enquête sur la manière dont les textes s'emparent des objets mécaniques, mettent en scène des objets mécaniques ou usent de la métaphore mécanique pour réfléchir sur l'engin et les artifices de la création technique mais aussi littéraire: l'écrivain se considère souvent lui aussi comme un artisan et le travail de la fiction pose des questions similaires de légitimité de la fabrication d'artifices imités de la nature.

On avait souhaité que l'enquête traverse autant que possible les genres les plus divers, de l'épopée à la lyrique en passant par le roman et le théâtre, les textes allégoriques ou les ouvrages scientifiques. J. Pavlevski s'est aventurée dans les sagas irlandaises médiévales et $\mathrm{D}$. Hüe a exploité le riche corpus de la poésie palinodique. C'est néanmoins le roman qui a focalisé l'essentiel des réflexions, et on peut le regretter. Plusieurs contributeurs ont toutefois exploré des textes savants ou à finalité pratique pour mieux saisir le traitement des machines qui apparaissent dans la littérature au regard de l'histoire des savoirs et savoir-faire: Denis Hüe avec les textes latins sur l'orgue, Myriam Clément pour le domaine de la pneumatique ou Véronique Dominguez avec les livres de secrets au théâtre. Robert Halleux, qui a ouvert notre séminaire ${ }^{14}$, nous a également proposé une mise au point précieuse.

Je tenterai, en partant d'une enquête lexicale qui souligne l'ambivalence sémantique des machines et engins, d'esquisser quelques axes de réflexion : les enquêtes sur différentes machines et engins ont montré comment les textes littéraires

13. Op. cit., p. 83 (chap. 9). Allusion à Gn 3,7.

14. $1^{\text {er }}$ octobre 2009. Spécialiste reconnu de l'histoire des sciences, des techniques et de l'industrie, de l'Antiquité à nos jours, il s'intéresse aux interactions entre savoirs scientifiques et savoirs techniques ainsi qu'aux origines des sciences appliquées, notamment à la métallurgie, mais aussi aux lapidaires et à l'alchimie. Voir R. Halleux, L'Histoire des sciences en Belgique des origines à 2000, Bruxelles, Crédit Communal, 1998 et Bruxelles, Dexia, La Renaissance du Livre, 2001; Cockerill. Deux siècles de technologie, Alleur, Éditions du Perron, 2002; Le Savoir de la main. Savants et artisans dans l'Europe préindustrielle, A. Colin, 2009. 
intègrent des savoirs mécaniques, tout en annexant à des finalités esthétiques et symboliques les caractéristiques techniques ou les connotations négatives rattachées aux arts mécaniques. Il est apparu que les machines et engins sont souvent le lieu de combinaisons et tensions variables entre mécanique et magique, dans une période où les catégories, classifications et hiérarchisations des arts et des sciences fluctuent. La figure de l'artifex, associée au clerc, à l'enchanteur et à la fée, semble particulièrement frappée d'un soupçon diabolique, ou à tout le moins, fréquemment dégradée. Art mécanique et art littéraire semblent l'un et l'autre peiner à acquérir une légitimité et à se débarrasser d'une ambiguïté morale qui pèse sur l'activité créatrice.

\section{Enquête lexicale}

Une petite enquête lexicale fournit un tremplin à la réflexion: le Dictionnaire Historique de la Langue Française (DHLF ${ }^{15}$ ) situe en 1377 l'apparition du mot machine en français, emprunté au latin machina qui hérite lui-même du grec makhana ${ }^{16}$. Une double connotation est possible dès le grec pour évoquer dans un registre positif le produit de l'invention et de l'ingéniosité technique, et par métonymie les objets qui en résultent: le mot peut aussi bien désigner dans un registre potentiellement péjoratif des objets ou moyens servant à tromper. Le verbe machiner au XIII ${ }^{\mathrm{e}}$ présente cette double valence, pouvant signifier "imaginer quelque chose d'ingénieux " mais aussi "ourdir et comploter », tandis que machination est spécialisée dans le registre péjoratif. À partir du XVII , les sens de ruse et d'artifice ingénieux auront vieilli et le mot se restreindra aux sens mécaniques avec les machines de guerre, à feu, puis à vapeur, à écrire ou à laver... Le sens strictement technique déjà présent en grec dans le domaine guerrier et théâtral ${ }^{17}$, puis en latin pour les engins de guerre ou destinés à soulever des charges, l'a donc emporté sur les sens médiévaux de " machine " qui pouvaient évoquer toute espèce de combinaison ou d'invention, potentiellement positive suscitant l'admiration ou inversement illusoire et trompeuse, éventuellement produit de la ruse.

15. Dictionnaire historique de la langue française, A. REY, Robert (dir.), 1992 (DHLF).

16. Il signifie à la fois invention, machination, et par métonymie, l'engin au sens technique notamment dans le domaine guerrier et théâtral. Il peut aussi bien qualifier la structure de l'univers, un ouvrage composé avec art ou des combinaisons ou inventions trompeuses.

17. Le domaine théâtral reste présent dans des dérivés comme " machiniste " (1634) ou "machinerie " (1881) pour désigner l' " ensemble des appareils utilisés pour mettre en place les décors » (DHLF). 
Le Dictionnaire du Moyen Français $\left(\mathrm{DMF}^{18}\right)$ révèle que l'emploi médiéval du mot est d'emblée métaphorique, pour désigner autre chose que des objets mécaniques au sens strict. En effet, l'apparition de machine s'observe « dans le syntagme machine corporelle avec la valeur analogique d'"ensemble d'éléments ayant la complexité d'une machine" 19 " chez Oresme vers 1377 :

Il me samble que par ce que je dirai a ces experiences, l'en pourroit respondre a toutes autres qui seroient amenees a ce propos. Et donques je met premierement que toute la machine corporelle ou toute la masse de tous les corps du monde est devisee en .II. parties. (DMF)

On retrouvera cet emploi de machine au sens d'assemblage des éléments de l'univers chez Descartes ou Pascal dans leurs réflexions sur les automatismes du corps. Le Moyen Âge hérite d'ailleurs de l'image de la machine céleste, attestée dans le Songe du vergiervers 1378 :

Et lez singuliers corps de la machine celeste sont ruillés dez singullieres intelligences qui lez muevent, et, ainsi, lez singuliers corps de la machine celeste si dependent dez singulieres intelligences, et lez singulieres intelligences si dependent de la primiere, et, ainsi, toute la machine celeste depent du Primier, qui est espirituel, qui a creé le ciel et la terre. (DMF)

Le DMF donne des exemples tardifs d'occurrences de "la machine du monde " ou de "la machine ronde " chez Robertet ou Simon de Phares ${ }^{20}$. La base Garnier ${ }^{21}$ permet de repérer la lexicalisation de « la machine ronde » dans les farces et de relever des attestations de « la machine du monde » chez Deschamps, Molinet ou dans le Vieil Testament.

La notion ambivalente d'ingéniosité associée au mot machine nous a amenés à l'associer à un autre mot médiéval aux sens tout aussi ambivalents: engin. R. Halleux a souligné que le mot machine était apparenté en grec à la métis, savoirfaire du constructeur ou de l'oiseleur, dont l'équivalent latin est ingenium, l'intelligence astucieuse et le produit de cette intelligence. Formé sur ingenium et la racine genius autour de la notion d'engendrer, il a pris en latin le sens de ruse tout en

18. Dictionnaire du Moyen Français, version 2010. ATILF CNRS - Nancy université. Site internet: http:// www.atilf.fr/dmf

19. Dictionnaire historique de la langue française, t. 2, p. 1160, col. 1.

20. Machine ronde: "Oncq Ulixes n'ot tel grace et faconde, N'autre qui soit soubz la machine ronde. " (Robertet cité par le DMF) « l'esclipse faicte le jour de la Passion de Nostre Saulveur et Redempteur Jhesu Crist à l'eure de midi n'estoit pas naturelle, ne le tremblement de terre, ne les autres choses merveilleuses qui advindrent, disant et publiant celui jour haultement ou qu'il failloit que le Dieu de nature souffrist, ou que toute la machine du monde se disolvist ». (Simon de Phares, cité par le DMF).

21. Garnier électronique (Corpus de la littérature narrative médiévale). 
pouvant signifier «talent " ou " invention habile ", et conserve ces sens en ancien français, où il peut désigner l'ingéniosité et ses manifestations concrètes, qu'elles soient positives ou stratagèmes destinés à tromper, et par métonymie divers instruments et machines, notamment les machines de guerre pouvant lancer de projectiles ou des dispositifs de vénerie destinés à piéger ou tuer des animaux. Les deux mots - machine et engin - peuvent d'ailleurs être coordonnés dans un contexte guerrier chez Bersuire:

Et se nous parlons de oevres si comme sont fossez, cloisons, engins et machines, qui [porroit] estre pareil aus rommains chevaliers? (DMF)

Les sens larges des deux mots nous ont donc autorisés à élargir l'investigation au-delà des seuls sens mécaniques.

\section{Carrefours et interactions entre arts mécaniques et imaginaire poétique}

On ne peut faire l'économie d'une remise en perspective historique sur les arts $\div$ mécaniques au Moyen Âge et même en amont. R. Halleux a souligné l'exploitation croissante au Moyen Âge de l'énergie de l'eau et du vent mais aussi l'importance des mécanismes de l'engrenage à angle droit, de l'arbre à came (généralisé au $\mathrm{XI}^{\mathrm{e}}$ siècle) ou de la combinaison bielle-manivelle pour transformer un mouvement alternatif en mouvement rotatif et inversement. Appliqués à différents domaines et matériaux, ces systèmes mécaniques ont renouvelé les techniques de martelage pour le fer, le papier ou le grain ou contribué à des innovations techniques remarquables comme la fonte ou le moulin à eau (généralisé au XII ${ }^{\mathrm{e}}$ siècle $^{22}$ ), souvent adoptées dans les monastères cisterciens. L'ingénierie militaire se complexifie également au XII ${ }^{\mathrm{e}}$ siècle avec des nouveautés dans les machines à balancier et contrepoids, les machines à ressorts ou les machines de siège ${ }^{23}$ qui permettent de gagner en précision et puissance. L'application des calculs géométriques complexes des techniciens arabes augmente les performances des machines à balancier ou contrepoids (perrière, bricole, mangonneau à roue de carrier, trébuchet de Villard de Honnecourt, couillard) ou des machines à ressort (arbalète à tour), qui perdurent à côté des bouches à feu et bombardes qui font leur apparition au début de la guerre de cent ans (vers 1324).

Le mot "ingeniator ", apparu en 1086 selon R. Halleux, renvoie à des architectes compétents dans des domaines aussi divers que les mesures, les engins de levage, l'art militaire, la pneumatique (mécanique des fluides) ou les automates. Si

22. Bernard et Marie-Claude Masson sont intervenus au CETM en janvier 2011 sur ce sujet.

23. Yves Simon nous avait présenté une synthèse au CETM en décembre 2009 sur ce sujet. 
le mot ingénieur ne s'impose qu'au XVI ${ }^{\mathrm{e}}$, engineor apparaît dès le XII ${ }^{\mathrm{e}} \mathrm{s}$. Villard de Honnecourt (vers 1230) et Léonard de Vinci, qui produisent des cahiers et catalogues d'ingénieurs, se rattachent à cette tradition mécanique, attestée jusqu'au $\mathrm{XVII}^{\mathrm{e}}$ siècle. C'est du côté des livres de secrets ou du seul livre de conduite de régisseurs conservé, celui de Mons, que V. Dominguez s'est tournée pour envisager les machines au théâtre, soulignant le rôle important des mystères dans l'élaboration des techniques de machines à la française. Elle observe d'ailleurs que ce sont les termes de secrets ou fainctes qui désignent les phénomènes spectaculaires mobilisant poulies, feu, galeries ou trappes, non le mot machine.

Les engins techniques sont assez peu présents dans les romans, comme si la technique semblait entrer en tension avec la possibilité du récit héroïque. Mais on trouve cependant des occurrences et mentions mécaniques parfois précises: C. Ferlampin, en s'intéressant à l'arbalète de Passelion dans Perceforest, note la présence de cette arme dévalorisée par rapport à l'épée mais qui a conquis une nouvelle place dans les conflits armés $\mathrm{du} \mathrm{Xv}^{\mathrm{e}}$ notamment du côté anglais. Décrite de manière précise avec sa clef qui déclenche le mécanisme et le coup mortel, elle apparaît dans ce roman tardif comme le lieu de conflits idéologiques entre plusieurs conceptions d'une chevalerie prise entre idéal et réalité. Le roman d'Escanor analysé par Myriam Clément présente un étonnant mécanisme de tuyauteries destiné à animer et faire chanter des oiseaux-automates qui met en ouvre des dispositifs pneumatiques.

Le cas des chars des sagas irlandaises, étudié par Joanna Pavlevski est particulièrement intéressant sur les approches disciplinaires et interdisciplinaires des machines: peut-on utiliser sans précautions de tels textes pour viser une reconstitution d'un référent historique en faisant l'économie des effets proprement littéraires, d'autant plus lorsqu'on a affaire à des strates de réécritures multiples? Autrement dit, comment appréhender les machines décrites dans ces textes entre realia et mirabilia, référent technique et jeu symbolique? Dans le cas de ces chars, il s'agit, dans une perspective archéologique, de savoir s'ils sont ceux de l'âge de fer, des chars romains, des chars insulaires ou une combinaison de ces modèles historiques avec des modèles intertextuels. Envisagés sous un angle littéraire, ces chars apportent des renseignements sur l'histoire des mentalités ou de l'ordre d'une anthropologie historique: ils participent en effet à la constitution de la figure du héros, en rapport métonymique avec son char, dont la possession manifeste une qualification guerrière et un statut d'exception, voire l'appartenance à l'autre monde. Ils s'inscrivent ainsi dans une logique de don ou d'investiture. Outre leur fonction identitaire, ils assurent une fonction axiologique entre féminin et masculin, à travers l'opposition de l'engin masculin et de l'engin féminin. Il y a donc un 
fonctionnement générique et symbolique des chars qui contribue à déformer le jeu de la référence. Dans un même souci méthodologique, R. Halleux a souligné comment il faut faire la part du savoir de la main et de l'œil ${ }^{24}$, qui va au-delà du savoir théorique, et prendre en compte les filtres et complexités du lexique technique. Le cas du mot " fertas » repéré par J. Pavlevski, oscillant entre la désignation du timon et du bras de suspension des chars dans les sagas irlandaises, témoigne des difficultés du vocabulaire technique, pour les auteurs ou copistes eux-mêmes. D. Hüe en s'intéressant à l'orgue part aussi d'une enquête lexicale, soulignant que sous le terme organum, il peut s'agir du grand instrument alimenté par des soufflets, mais aussi de toute machinerie complexe ou même de musique polyphonique. M. Clément intègre de même la dimension lexicale à son enquête.

Plusieurs contributeurs se sont intéressés à la manière dont la littérature s'approprie des objets mécaniques en les annexant à des fins esthétiques et/ou proprement littéraires, à travers leur potentiel symbolique ou métaphorique, y compris métatextuel.

D. Hüe a montré comment l'orgue, instrument à vent et machine complexe, soutient une réflexion sur le spiritus et l'harmonie universelle. Instrument liturgique et objet d'une fabrication savante, il produit un chant de grâce. Chez les poètes du puy de Rouen, il peut devenir métaphore de Marie, allégorie de la circulation du souffle et du vent de l'esprit. C. Ferlampin-Acher a souligné comment l'arbalète de Passelion dans le Perceforest, arme de technologie relativement récente et opposée à l'épée, oscille entre le statut de realia avec le détail technique de la clef et de motif merveilleux, pour caractériser un héros hors du commun: attribut merveilleux d'un jeune héros né avec son arbalète à la main, elle est associée à la vitalité et même à une sexualité reproductive heureuse; elle est en même temps utilisée comme une arme de chasse et l'outil d'un jeu enfantin et sportif qui prend l'adversaire pour cible, non sans allusion possible aux concours organisés par les confréries. L'arbalète est ainsi intégrée dans une conjointure romanesque signifiante, comme arme d'une vengeance annoncée et différée, élément d'un jeu civilisateur, mais aussi comme arme humoristique puisque c'est un enfant de dix mois qui s'en sert.

La machine, comme les objets en général, offre aussi une ressource possible à l'esthétique allégorique par le jeu de la description métonymique ou synecdochique. Ainsi dans Le Chastel périlleux de frère Robert, on rencontre quatre

24. Voir Robert Halleux, Le Savoir de la main. Savants et artisans dans l'Europe pré-industrielle, A. Colin, 2009. 
" arbalestes " et quatre "mangonnaux " associés à des dispositions de l'âme ${ }^{25}$, dans le dispositif de défense dont l'âme-château doit se doter. Michèle Gally avait étudié dans un programme précédent du CETM le cas de l'horloge annexée par Froissart dans L'Horloge amoureuse à une réflexion sur l'expérience amoureuse et sur la lyrique ${ }^{26}$.

Les automates romanesques ont reçu une attention particulière en faisant l'objet de quatre réflexions complémentaires mais finalement assez différentes. Ces machines qui entretiennent un rapport privilégié aux merveilles orientales mais comptent aussi parmi les merveilles arthuriennes ont en effet connu un grand succès dans les romans médiévaux.

Hélène Bouget a examiné le traitement de la machine et des automates dans le Perlesvaus comme révélateur d'une esthétique romanesque: le mécanisme, entre rationalité et merveilleux diabolique, description et énigme, technique et féérique, est le signe de tensions idéologiques et esthétiques dans un roman qui peine à se construire comme roman de la matière arthurienne. L'imprimé du XVI ${ }^{\mathrm{e}}$ et la version galloise sont des symptômes supplémentaires de cette hétérogénéité. Associés au monde antique et byzantin, les automates sont, comme l'a souligné Chantal Connochie-Bourgne les instruments d'une conquête amoureuse sous la forme du cheval de fust (bois), dans Cléomadès, non sans lien avec le monde nocturne d'une sexualité à maîtriser, alors qu'ils ne sont, sous la forme des gardiens maléfiques et diaboliques du Perlesvaus que des signaux d'une épreuve élective qui se joue en dehors d'eux. M. Clément montre comment les imaginaires du poète et du mécanicien se rencontrent dans la scénarisation des oiseaux automates chanteurs du roman d'Escanor et comment le succès des automates orientaux à partir du XIII ${ }^{\mathrm{e}}$ en Occident a pu transiter par la Sicile. Les oiseaux-automates n'incarnent plus la figure d'un souverain tout puissant et démiurge, qui prolonge la création par la fabrication d'objets imitant la nature; ce ne sont plus des objets de contemplation, mais des objets de manipulation au service d'une expérience ludique de l'usager, à la manière des machinations participatives du parc de Hesdin. De manière originale, c'est peut-être la figure du lecteur de roman qui est ainsi repré-

25. A critical édition and study of frère Robert (Chartreux). Le Chastel périlleux by Sister Marie Brion, Analeta Cartusiana 19, 2 vol., Institut für Englische Sprache und Literatur, Universität Salsburg, 1974. Considération de sa propre orine ou naissance, Ignorance de son propre estat, Considération de la mort et Attente du grand jugement pour les quatre arbalettes, p. 323. Pour les mangonnaux, voir p. 344.

26. "Sens et enjeux d'une nouvelle métaphore: L'Horloge amoureus de Froissart ", dans Cloches et horloges dans les textes médiévaux. Mesurer et maîtriser le temps, Rennes, Presses Universitaires de Rennes, Interférences, 2012, p. 261-275. 
sentée et invitée à une expérience de divertissement. C'est donc la conception même de l'activité esthétique, et plus particulièrement romanesque, qui se dit à travers l'ekphrasis de l'automate. A. Berthelot quant à elle souligne comment à travers les automates romanesques se manifeste un " entre-deux sémiotique ", magie et technique restant fortement imbriquées, et postulant ainsi un statut flottant de l'artifex.

\section{Ingéniosité et illusion : une réflexion sur la création artistique et les figures d'artifex}

La machine, comme produit d'une ingéniosité, suscite l'étonnement et la fascination, mais non sans inquiétude sur ses origines ou contaminations potentiellement diaboliques. En effet, si la machine génère une admiration et une curiosité, c'est qu'elle semble dotée de pouvoirs d'autonomie, voire d'un fonctionnement perpétuel en dehors de l'impulsion humaine. En ce sens, elle tend à se faire mimesis et imitation ou illusion de réalité, mise en oeuvre par un maître des " engins".

Merlin, dans les ambiguïtés de sa naissance et de ses pouvoirs manifeste cette ambivalence de l'engin comme ingéniosité, tout comme les accointances fréquentes observées entre technique et magie, technique et diabolique, que Karin Ueltschi et Anne Berthelot ont pointé dans les figures de Virgile ou d'Aroès, et C. Connochie dans l'inventeur hideux et pervers du cheval de fust qu' est Crompart. A. Berthelot et K. Ueltshi montrent que les catégories ne sont pas étanches entre magie et technique: au contraire, elles se combinent et se complètent selon des modalités fluctuantes. À travers un Virgile à la fois poète, savant, clerc, magicien et progressivement détérioré en nigromancien, K. Ueltschi observe les classements instables des domaines du savoir et des disciplines scientifiques. Dans l'opposition entre domaine intellectuel et manuel, les arts mécaniques distingués des arts libéraux restent toujours un peu suspects, malgré l'éloge qu'en font certains auteurs. Ce n'est qu’à partir de la Renaissance que science et magie se sépareront plus nettement.

J'ai pour ma part relevé dans Amadas et Ydoine le paradigme des machinistes que sont Ydoine, les fées, Fortune et le narrateur comme autant de maitres des destins et des illusions. Ydoine, à la fois fabulatrice, metteur(e) en scène et actrice, maîtrise ainsi les ruses de la fiction, de la magie et du théâtre tandis que la roue de Fortune fournit l'emblème d'une mécanique narrative du renversement perpétuel. C. Connochie remarque de son côté que le cheval de fust dans Cléomadès, doté de chevilles pour sa conduite, représente l'instrument romanesque du romancier. C'est l'art de la fiction qui est ainsi interrogé, avec ses ambivalences morales et 
son potentiel diabolique. Au service d'un code de fiction, les machines et engins au théâtre posent cette même question de l'illusion et interrogent sa compatibilité avec la dévotion. V. Dominguez a ainsi souligné la fonction des machines et feintes théâtrales comme "socle et caution d'un théâtre de l'illusion consentie " et « laboratoires de l'illusion».

Machines et engins médiévaux cristallisent donc une réflexivité sur l'art littéraire. La métaphore de la machine ou de l'engin est utilisée par la littérature pour représenter son propre fonctionnement, notamment l'invention romanesque. Cette métaphore mécanique se retrouve dans la "Machine-littérature " de Calvino ${ }^{27}$ qui imagine remplacer l'écrivain par une machine pour produire une littérature qui serait combinaison de mécanismes et jeu réglé. On la retrouve encore chez Barthes qui voit dans le livre sous la forme du dictionnaire une "machine à rêver ${ }^{28}$ ". Les critiques usent aussi volontiers de la métaphore de la machine pour qualifier une ouvre: ainsi B. Rey-Flaud présente la farce comme " une machine à rire ${ }^{29}$ dont les personnages sont tous potentiellement victimes.

Entre la vieille métaphore du monde-machine, attestée dans la traduction de la Consolation de Philosophie de Boèce et l'archétypale roue de Fortune dont La Machine infernale de Cocteau est un avatar $\operatorname{tardif}{ }^{30}$, la machine semble figurer des mécanismes dont la maîtrise échappe à l'homme et revient à des instances qui le transcendent. Envisager la production littéraire comme machine reviendrait alors à déresponsabiliser (ou du moins tenter de déresponsabiliser) son auteur, en plaçant le principe de création dynamique dans un ailleurs ou une logique extérieure, comme pour dédouaner ou feindre de dédouaner l'auteur d'un rôle de créateur, et simultanément, penser le créateur comme machiniste ambivalent.

R. Halleux avait observé au début de notre séminaire que les préoccupations des ingénieurs rejoignent significativement celles des écrivains lorsqu'ils envisagent d'organiser la science des machines comme une syntaxe ou un langage, lorsqu' ils travaillent sur un principe de mimesis ou se laissent aller à l'imaginaire en oubliant les contraintes techniques, dans un enthousiasme confiant dans les possibilités de l'art. C'est en effet le pouvoir de création qu'interrogent les machines, en même

27. Italo Calvino, La Machine-littérature, Paris, Le Seuil, 1993.

28. Préface du dictionnaire Hachette, R. Barthes, Euvres complètes, vol. 5 (1977-1980), édition établie et présentée par Éric Marty, Paris, Le Seuil, 2002, p. 924.

29. Bernadette Rey-Flaud, La Farce ou la machine à rire. Théorie d'un genre dramatique (1450-1550), Genève, Droz, 1984.

30. «Regarde, spectateur, remontée à bloc, de telle sorte que le ressort se déroule avec lenteur tout le long d'une vie humaine, une des plus parfaites machines construites par les dieux infernaux pour l'anéantissement mathématique d'un mortel. ", Jean Cocteau, La Machine infernale, acte 1. 
temps que le rapport de l'homme au monde, qui engage les dimensions du savoir et du pouvoir mais aussi un positionnement d'ordre éthique et esthétique.

Les nouveaux écrans et outils de communication ou les drones ne manifestent-ils pas aujourd'hui cette faculté des machines à bouleverser notre rapport au monde? Les écrans numériques modifient notre rapport à l'espace, au temps et à autrui, happant l'homme lui-même dans le virtuel selon Jean Baudrillard ${ }^{31}$. $\mathrm{La}$ " théorie du drone » de Grégoire Chamayou ${ }^{32}$ offre aussi une analyse passionnante: ce philosophe observe que l'on a affaire avec les drones à « des machinesscribes, des greffiers volants et robotisés qui dresseraient en temps réels le procèsverbal des moindres activités du monde situé en contrebas " (p. 62), et que ces outils d'un État chasseur développent un terrorisme d'état. Doté d'yeux, d'oreilles et de bras, ils manifestent un fantasme d'omniscience et d'omnipotence et ont un effet pétrifiant sur leurs cibles quand ils ne sont pas purement et simplement un moyen d' "exécution extra-judiciaire " (p. 87). Autour d'une technologie militaire, ce sont des questions éthiques, psychologiques et juridiques qui sont posées, où l'on retrouve en creux l'interrogation médiévale sur les potentialités, sinon diaboliques, du moins négatives de la machine et de l’ingéniosité du machiniste.

31. Voir Le Système des objets: la consommation des signes, éd. Gallimard, Paris, 1978 ou http://www.egs. edu/faculty/jean-baudrillard/articles/ecran-total/ (consulté le 26.05.2014) : "À un certain niveau de machination, d'immersion dans la machinerie virtuelle, il n'y a plus de distinction homme/machine: la machine est des deux côtés de l'interface. Peut-être même n'êtes-vous plus que son espace à elle -l'homme devenu la réalité virtuelle de la machine, son opérateur en miroir. " "Ainsi l'attraction de toutes ces machines virtuelles vient sans doute moins de la soif d'information et de connaissance, ni même de rencontre, que du désir de disparition, et de la possibilité de se dissoudre dans une convivialité fantôme. » Voir aussi : http://www.watsoninstitute.org/infopeace/vy2k/baudrillard.cfm. "Toutes nos machines sont des écrans, nous-mêmes sommes devenus écrans, et l'interactivité des hommes est devenue celle des écrans. Rien de ce qui s'inscrit sur les écrans n'est fait pour être déchiffré en profondeur, mais bien pour être exploré instantanément, dans une réaction immédiate au sens, dans une circonvolution immédiate de pôles de la représentation. "

32. Théorie du drone, La fabrique éditions, Paris, 2013. 